\title{
Synchrotron radiation and absence of linear polarization in the colliding wind binary WR 146
}

\author{
C. A. Hales ${ }^{1}$, P. Benaglia ${ }^{2,3}$, S. del Palacio ${ }^{2,3, \star}$, G. E. Romero ${ }^{2,3}$, and B. S. Koribalski ${ }^{4}$ \\ 1 National Radio Astronomy Observatory, PO Box 0, Socorro, NM 87801, USA \\ e-mail: chales@nrao.edu \\ 2 Instituto Argentino de Radioastronomía, CCT-La Plata, CONICET, Argentina \\ 3 Facultad de Ciencias Astronomicas y Geofisicas, Universidad Nacional de La Plata, Pasea del Bosque s/n, 1900 La Plata, Argentina \\ 4 Australia Telescope National Facility, CSIRO Astronomy \& Space Science, PO Box 76, Epping, NSW 1710, Australia
}

Received 2 September 2016 / Accepted 21 October 2016

\begin{abstract}
Context. Several massive early-type binaries exhibit non-thermal emission which has been attributed to synchrotron radiation from particles accelerated by diffusive shock acceleration (DSA) in the wind-collision region (WCR). If the magnetic field in the strong shocks is ordered, its component parallel to the shock front should be enhanced, and the resultant synchrotron radiation would be polarized. However, such polarization has never been measured.

Aims. We aim to determine the percentage of linearly polarized emission from the well-known non-thermal radio emitter WR 146, a WC6+O8 system.

Methods. We performed spatially-unresolved radio continuum observations of WR 146 at $5 \mathrm{~cm}$ and $20 \mathrm{~cm}$ with the Karl G. Jansky Very Large Array. We constructed a numerical model to investigate a scenario where particles are accelerated by turbulent magnetic reconnection (MR), and we performed a quantitative analysis of possible depolarization effects.

Results. No linearly polarized radio emission was detected. The data constrain the fractional linear polarization to less than $0.6 \%$ between 1 to $8 \mathrm{GHz}$. This is compatible with a high level of turbulence and a dominant random component in the magnetic field. In this case the relativistic particles could be produced by turbulent magnetic reconnection. In order for this scenario to satisfy the required non-thermal energy budget, the strength of the magnetic field in the WCR must be as high as $\sim 150$ mG. However, if the magnetic field is ordered and DSA is ongoing, then a combination of internal and external Faraday rotation could equally account for the depolarization of the emission.

Conclusions. The absence of polarization could be caused by a highly turbulent magnetic field, other depolarization mechanisms such as Faraday rotation in the stellar wind, or a combination of these processes. It is not clear whether it is possible to develop the high level of turbulence and strong magnetic fields required for efficient MR in a long-period binary such as WR 146. This scenario might also have trouble explaining the low-frequency cutoff in the spectrum. We therefore favor a scenario where particles are accelerated through DSA and the depolarization is produced by mechanisms other than a large ratio between random to regular magnetic fields.
\end{abstract}

Key words. polarization - radio continuum: stars - radiation mechanisms: non-thermal - stars: individual: WR 146 stars: winds, outflows

\section{Introduction}

Hot, early-type stars (O-B2, Wolf-Rayet) are commonly found in stellar systems. If the two components of the system exhibit strong winds, these can interact forming a wind collision region (WCR); we refer to such systems as colliding-wind binaries (CWBs). Colliding stellar winds are clearly detected at a few $\mathrm{GHz}$ using radio interferometers, as methodical studies such as Bieging et al. (1989) show. Observations of these systems over a range of radio frequencies show flux density decreasing with the observing frequency, something characteristic of non-thermal (NT) radiation. The recent catalog of particleaccelerating colliding-wind binaries (PACWB) compiled by De Becker \& Raucq (2013) presents very detailed information of what is known about these systems. Their emission is interpreted as having a synchrotron origin (i.e. due to relativistic electrons moving in the presence of a magnetic field). Synchrotron radiation is expected to be polarized. However, to date there are no reports of polarized radio emission from a PACWB.

\footnotetext{
$\star$ Fellow of CONICET.
}

According to the PACWB catalog there are more than 40 known binary systems capable of producing synchrotron radiation. One of these is WR 146, the brightest WR star at radio wavelengths and the fourth to be associated with a NT radio source (Felli \& Massi 1991). It is located at a distance of $1.2 \mathrm{kpc}$ and is thought to be a WC6+O8 system (Niemela et al. 1998). MERLIN observations at $5 \mathrm{GHz}$ (Dougherty et al. 1996) show a NT source located north of the primary star. The system was observed in 2004 with the VLA in combination with the VLBA Pie Town antenna, from 1.4 to $43 \mathrm{GHz}$ (O'Connor et al. 2005). The $43 \mathrm{GHz}$ observations reveal two components, separated by about 0.2 arcsec: a northern source coincident with the secondary star and a southern source coincident with the primary star. The $1.4 \mathrm{GHz}$ observations reveal that the NT source is located part-way between the two components, close to the northern one. Only the NT source is detected at the lower frequencies $(1.4 \mathrm{GHz}, 5 \mathrm{GHz})$. The system was monitored over $10 \mathrm{yr}$ with the WSRT at $350 \mathrm{MHz}$ and $1.4 \mathrm{GHz}$. Variability with period $3.38 \mathrm{yr}$ suggests the presence of a third component that modulates the O star wind (Setia Gunawan et al. 2000). 
The polarization characteristics of a synchrotron source can potentially provide useful and unique information about physical conditions within the source (Cioffi \& Jones 1980). An accurate determination of the polarization degree (PD) allows one, in principle, to distinguish among different scenarios. If the observed PD is between a few percent up to a few tens, this would provide decisive evidence of the synchrotron origin of the NT emission and constrain the degree of turbulence in the plasma. If an extremely high polarization (above 70\%) is detected, the underlying physics of particle acceleration in CWBs will be shown to be different from what has been traditionally assumed in most theoretical models. Finally, if no polarization is detected (i.e., PD $\lesssim 1 \%$ ), the main acceleration and emission mechanisms of the standard model of CWBs - diffusive shock acceleration (DSA) and synchrotron radiation, respectively might need to be reconsidered. Therefore, polarization measurements can improve our knowledge of CWBs.

This work presents the first radio polarimetric study of a CWB, focusing on WR 146. We describe our observations and data reduction in Sect. 2. Results are presented in Sect. 3. In Sect. 4 we discuss acceleration processes and interpret our polarization findings. Concluding remarks and directions for future research are presented in Sect. 5.

\section{Observations and data reduction}

We observed WR 146 with the Karl G. Jansky Very Large Array (VLA) on 2015 May 4 under Project Code 15A-480. The array was in B configuration. Full polarization observations were performed in two frequency bands. These were centered at $1.5 \mathrm{GHz}$ (20 cm, $L$-band) and $6 \mathrm{GHz}(5 \mathrm{~cm}, C$-band) spanning bandwidths of $1 \mathrm{GHz}$ and $4 \mathrm{GHz}$, respectively. In the $20 \mathrm{~cm}$ band, the correlator was configured to deliver 16 spectral windows with $64 \times 1 \mathrm{MHz}$ channels. The $5 \mathrm{~cm}$ band was configured to deliver 32 spectral windows with $64 \times 2 \mathrm{MHz}$ channels. The time sampling was $3 \mathrm{~s}$ for both bands. The total observing time on WR 146 was 19 min at $L$-band and 14 min at $C$-band. We observed 3C 138 for the purpose of flux density and position angle calibration, J2007+4029 for amplitude and phase calibration, and the unpolarized source $\mathbf{J} 2355+4950$ for instrumental leakage calibration. $\mathrm{J} 2355+4950$ is less than $0.25 \%$ polarized at $L$-band and $C$-band.

The data were reduced using version 4.3 .0 of the CASA package (McMullin et al. 2007). Hanning smoothing was performed. RFI was identified manually and conservatively flagged. The flux density scale was referenced to the most recent 2012 value for 3C 138 from Perley \& Butler (2013a). The position angle of $3 \mathrm{C} 138$ was assumed to be $-11^{\circ}$ on all spatial scales across $L$-band and $C$-band. This is within a few degrees of the values presented by Perley \& Butler (2013b) based on D configuration observations. However, their values are only loosely appropriate for translation to our B configuration data because 3C 138 exhibits polarization structure on sub-arcsecond scales (Cotton et al. 1997). We conservatively estimate that any intrinsic rotation measure (RM) left unaccounted in our model of $3 \mathrm{C} 138$ is no worse than $2 \mathrm{rad} \mathrm{m}^{-2}$. No dedicated corrections were performed to account for atmospheric Faraday rotation taking place within the ionosphere and plasmasphere (the necessary CASA functionality contained an error in version 4.3.0, so it was not used). Line of sight atmospheric RMs throughout our observation were approximately constant at $\sim 6 \mathrm{rad} \mathrm{m}^{-2}$, estimated by combining GPS-derived total electron content data from the International GNSS Service with the International Geomagnetic Reference Field model of the Earth's magnetic field. Without correction, this atmospheric RM was absorbed into our position angle calibration, imparting a residual $\sim 1 \mathrm{rad} \mathrm{m}^{-2}$ on our target data. Combining these two uncertainties, we estimate that our WR 146 data are affected by a systematic RM of no more than approximately $2 \mathrm{rad} \mathrm{m}^{-2}$. This is negligible given our resolution in Faraday depth space, described below. We performed one round of phase self-calibration on WR 146 in each band.

The total intensity data were imaged per band as well as per spectral window, the latter every $64 \mathrm{MHz}$ in the $20 \mathrm{~cm}$ band (minus 3 windows affected by RFI) and every $128 \mathrm{MHz}$ in the $5 \mathrm{~cm}$ band (minus 4 windows affected by RFI). The continuum rms noises in the full-band $20 \mathrm{~cm}$ and $5 \mathrm{~cm}$ images are $40 \mu \mathrm{Jy}$ beam $^{-1}$ and $12 \mu \mathrm{Jy}$ beam $^{-1}$, respectively. These values are worse than theoretical expectations (almost double) due to unusually disruptive RFI. However, taking into account observational contingencies, the sensitivities are sufficient for the objectives in this work. The spatial resolutions in the full-band $20 \mathrm{~cm}$ and $5 \mathrm{~cm}$ images are $\sim 4^{\prime \prime}$ and $\sim 1^{\prime \prime}$, respectively. Flux density measurements in the full-band and per spectral window images were performed using BLOBCAT (Hales et al. 2012b).

We imaged the Stokes $Q$ and $U$ data by pre-averaging every 2 input spectral channels to form an output image cube with $2 \mathrm{MHz}$ channels across $L$-band and $4 \mathrm{MHz}$ channels across $C$-band. Each image plane was then smoothed to a common spatial resolution of $14^{\prime \prime} \times 6^{\prime \prime}$, set by the lowest frequency. Using this data we performed RM synthesis (Brentjens \& de Bruyn 2005). We examined the data in four different frequency ranges: the combined 1-2 GHz and 4-8 GHz data, and subsets $1-2 \mathrm{GHz}$, 4-8 GHz, and 6-8 GHz. The resolution in Faraday depth space for each of these frequency ranges is approximately $40 \mathrm{rad} \mathrm{m}^{-2}$, $50 \mathrm{rad} \mathrm{m}^{-2}, 900 \mathrm{rad} \mathrm{m}^{-2}$, and $3000 \mathrm{rad} \mathrm{m}^{-2}$, respectively. The effective rms noise ( $\sigma_{Q U}$; Hales et al. 2012a) for each of the respective ranges is $22 \mu \mathrm{Jy}$ beam $^{-1}, 30 \mu \mathrm{Jy} \mathrm{beam}{ }^{-1}$, $34 \mu \mathrm{Jy}$ beam $^{-1}$, and $51 \mu \mathrm{Jy}$ beam $^{-1}$.

\section{Results}

We detect WR 146 as a single spatially-unresolved source in the $20 \mathrm{~cm}$ and $5 \mathrm{~cm}$ total intensity images. The flux density is $79.8 \pm 1.6 \mathrm{mJy}$ at $1.52 \mathrm{GHz}$ and $35.7 \pm 0.7 \mathrm{mJy}$ at $6.00 \mathrm{GHz}$, obtained from the full-band images. The errors include a systematic absolute flux density uncertainty of $2 \%$ added in quadrature to the statistical errors previously quoted.

The spectrum obtained from the per spectral window data is displayed in Fig. 1 and tabulated in the appendix. The figure shows marginal evidence for a turnover below $1.5 \mathrm{GHz}$. The spectral index ( $\alpha$ such that $S_{v} \propto v^{\alpha}$ ) is $-0.58 \pm 0.02$ when fit to the flux densities from all spectral windows. It is possible that the flux densities at the lower frequencies are affected by absorption. To account for this, we fit only the higher frequency data from spectral windows within the $5 \mathrm{~cm}$ band, obtaining a softer value of $-0.62 \pm 0.02$ (Fig. 1). To highlight the putative turnover, in Fig. 1 (and later in Fig. 2) we also plot measurements at $327 \mathrm{MHz}$ from Benaglia \& Ishwara-Chandra (priv. comm.; observed with GMRT in Oct. 2014) and Taylor et al. (1996), where the cutoff becomes more pronounced. Note, however, that their observations are not contemporaneous with those presented in this work, so their data points are not expected to correspond with our spectral fit.

We do not detect any linearly polarized emission from WR 146 within the 4 frequency ranges examined. Upper bounds ${ }^{1}$ are

\footnotetext{
1 Adhering to the terminology regarding bound versus limit from Kashyap et al. (2010).
} 


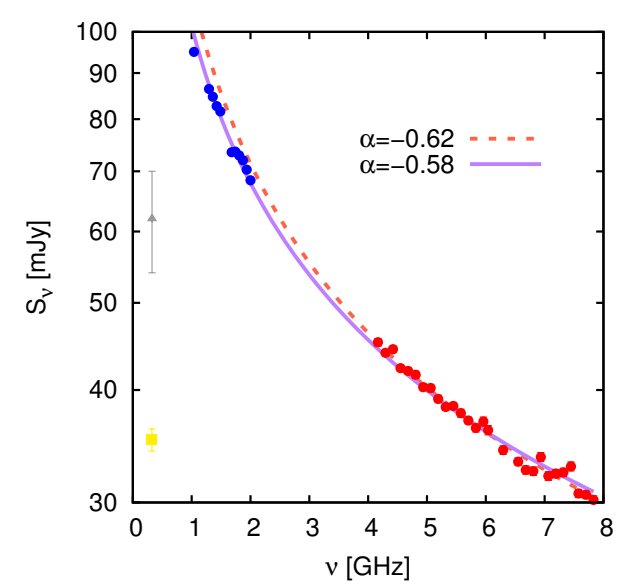

Fig. 1. Radio observations at $L$-band (blue points) and $C$-band (red points). We show the spectral fit $S_{v} \propto v^{\alpha}$ to the whole data set (solid purple line) and only to the $C$-band data (dashed orange line). We also show the flux densities measured at $327 \mathrm{MHz}$ by Taylor et al. (1996) (grey triangle) and Benaglia \& Ishwara-Chandra (priv. comm., observed in Oct. 2014, yellow square).

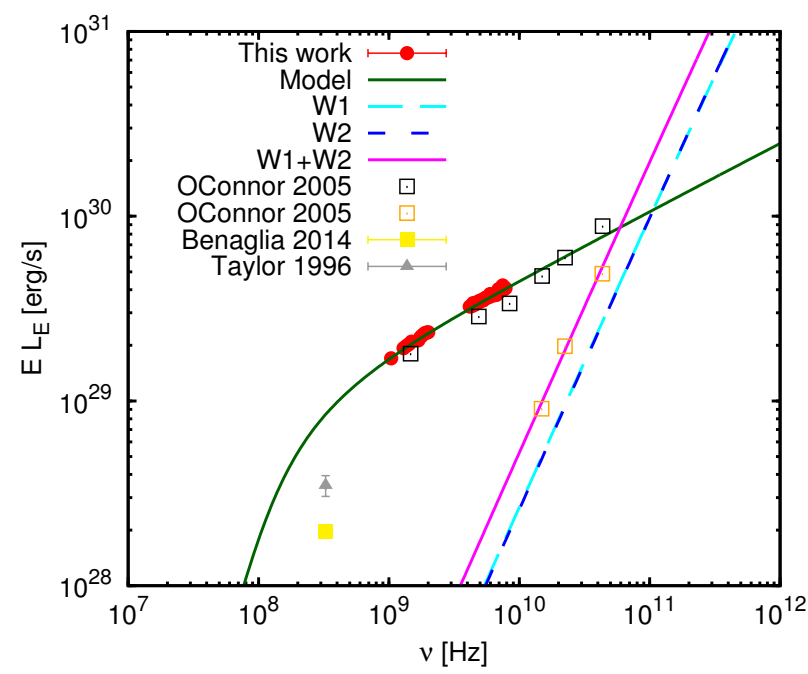

Fig. 2. Luminosities in the $20 \mathrm{~cm}$ and $5 \mathrm{~cm}$ bands from our per spectral window data (red points). The solid green curve is the simulated emission considering acceleration by MR, and synchrotron emission suffering free-free absorption in the stellar wind. We also include data points from Taylor et al. (1996) (grey triangle) and Benaglia \& IshwaraChandra (priv. comm., observed in Oct. 2014, yellow circle), and from O'Connor et al. (2005) for the total flux density (dark grey squares) and the WR emission (orange squares). The straight lines represent the separated (dashed) and summed (solid) free-free emission from the winds using Eq. (8) from Wright \& Barlow (1975).

reported in Table 1. In each case, the statistical significance is below the Gaussian equivalent of a 4 sigma detection (Hales et al. 2012a). Combining these results with total intensity flux densities, the PD at $L$-band is less than $0.15 \%(<120 \mu \mathrm{Jy} / 80 \mathrm{mJy})$, at $C$-band it is less than $0.5 \%(<180 \mu \mathrm{Jy} / 36 \mathrm{mJy})$, and within the upper half of $C$-band $(6-8 \mathrm{GHz})$ it is less than $0.6 \%$ $(<190 \mu \mathrm{Jy} / 32 \mathrm{mJy})$. Thus overall, the WR 146 emission exhibits less than $0.6 \%$ fractional polarization across $1-8 \mathrm{GHz}$.

\section{Discussion}

Since the pioneering studies of Eichler \& Usov (1993), the NT radio emission of PACWBs has been explained as synchrotron
Table 1. Upper bounds on the linearly polarized flux density (LPFD) for different portions of the data.

\begin{tabular}{ccc}
\hline \hline Data & FD range & LPFD \\
\hline $1-2,4-8 \mathrm{GHz}$ & $\pm 2 \times 10^{6} \mathrm{rad} \mathrm{m}^{-2}$ & $<140 \mu \mathrm{Jy}$ \\
$1-2 \mathrm{GHz}$ & $\pm 4 \times 10^{4} \mathrm{rad} \mathrm{m}^{-2}$ & $<120 \mu \mathrm{Jy}$ \\
$4-8 \mathrm{GHz}$ & $\pm 10^{6} \mathrm{rad} \mathrm{m}^{-2}$ & $<180 \mu \mathrm{Jy}$ \\
$6-8 \mathrm{GHz}$ & $\pm 10^{6} \mathrm{rad} \mathrm{m}^{-2}$ & $<190 \mu \mathrm{Jy}$ \\
\hline
\end{tabular}

Notes. The bounds give the peak in the Faraday depth (FD) spectrum over the FD range examined using RM synthesis.

radiation produced by a population of relativistic electrons interacting with the local magnetic field. It has been suggested that the most suitable mechanism to account for the acceleration of electrons in PACWBs is DSA through a first-order Fermi process (Romero et al. 1999; Benaglia \& Romero 2003; Pittard \& Dougherty 2006; Reitberger et al. 2014), although magnetic reconnection (MR) is also possible (Jardine et al. 1996). For DSA to operate efficiently, a strong shock, a turbulent motion of the plasma, and a disordered magnetic field are required. The plasma is expected to develop instabilities near the contact discontinuity (Stevens et al. 1992), which would provide the required turbulence for this scenario, although a very high turbulence might debilitate the shock and therefore reduce the efficiency of DSA. On the other hand, for MR to be efficient, small-scale turbulent motions are necessary (Lazarian \& Vishniac 1999). Hence, this mechanism cannot be discarded in a very turbulent regime (Falceta-Gonçalves \& Abraham 2012). The magnetic field is not required to be high for DSA (Bosch-Ramon \& Rieger 2012), in contrast to the case of MR.

In any case, the high-energy electrons are expected to obtain a power-law distribution in energy with a spectral index $p \sim 2$. The resultant synchrotron radiation is intrinsically polarized up to a value of $\Pi_{i}(p) \sim 70 \%$. However, a turbulent magnetic field greatly decreases the PD. If the random component of the field is $B_{\mathrm{r}}$ and the ordered field is $B_{0}$, the observed PD becomes (Burn 1966):

$\Pi_{\mathrm{obs}}(p)=\Pi_{i}(p) \frac{B_{0}^{2}}{B_{0}^{2}+B_{\mathrm{r}}^{2}} \xi(\lambda)$,

where the function $\xi(\lambda)$ takes into account possible depolarization effects (wavelength-dependent in some cases; Sect. 4.2). Since the magnetic field lines are frozen in the plasma, the random component of the magnetic field is linked to the degree of turbulence in the WCR, measured by the ratio $B_{\mathrm{r}} / B_{0}$. Therefore, the PD is strictly related to the degree of turbulence, and a small polarization could be indirect evidence of a highly turbulent medium. Considering solely this effect, our result of nondetection of polarization in WR 146 points to a very high ratio $B_{\mathrm{r}} / B_{0}>8$. DSA is not expected to be efficient under such conditions due to the consequently weaker shocks, which leads to the conclusion that MR could be the principal acceleration mechanism acting on this PACWB.

\subsection{Turbulent magnetic reconnection}

MR is a process that develops when two converging flows meet. If the magnetic fields carried by the flows have opposite 
polarity, the magnetic field lines reconnect and magnetic energy is released. This energy can be converted to kinetic energy, i.e., sheating and/or particle acceleration, in multiple small-scale MR events that develop in turbulent plasmas. In such case particles are accelerated in multiple magnetic islands (Kowal et al. 2011). The acceleration timescale is $t_{\mathrm{acc}}^{-1}=$ $\eta_{\text {acc }} e c B / E$, where $\eta_{\text {acc }} \sim 0.3\left(v_{\text {rec }} / c\right)^{2}$ is the acceleration efficiency, $v_{\text {rec }}$ the reconnection velocity, $e$ and $E$ the charge and energy of the particle, respectively, and $B$ the ambient magnetic field. Depending on the conditions in the plasma, the spectral index can be $p<2.5$ (Drury 2012), which is in agreement with the injection spectral index derived from our observations, $p=-2 \alpha+1 \simeq 2.3 \pm 0.1$.

We applied the NT emission model described in del Palacio et al. (2016) to test whether turbulent MR can account for the accelerated particles in WR 146. This model considers the adiabatic wind shocks to be thin enough to neglect their width, and that the relativistic particles are attached to the flow streamlines through the chaotic $B$-component. The NT particle content is modeled as sets of linear-emitters. In each linear-emitter, a population of relativistic particles is injected at a given location with a distribution $Q(E) \propto E^{-p}$, and evolve until reaching the stationary particle distribution at positions along the streamline. The relevant energy losses (IC scattering and synchrotron for electrons; $p$-p interactions for protons) and the advection of particles are taken into account when computing the particle evolution. The adopted system parameters are summarized in Table 2. The adopted parameters yield a linear distance of $D \sim 200 \mathrm{AU}$. The small value of $\eta$ means that the shocked structure is much closer to the secondary star.

The WCR consists of the shocked stellar wind from the primary (S1) and the shocked stellar wind from the secondary (S2), each with different properties. Magnetic field notation is as follows: $B_{* 1}$ and $B_{* 2}$ are the stellar surface magnetic fields, and $B_{1}$ and $B_{2}$ are the compressed fields in the shocks S1 and S2 which are (in our simple model) the WCR. In both shocks we considered a reconnection velocity given by $v_{\text {rec }}=0.6 v_{\mathrm{A}}$ (del Valle \& Romero 2012), where $v_{\mathrm{A}}$ is the Alfvén velocity. We also assumed that $\sim 4 \%$ of the released magnetic energy goes to accelerating electrons (del Valle et al. 2011, and references therein).

Under the mentioned conditions, our results show that as long as the WR star is not extremely magnetized (surface magnetic field $B_{* 1}<2000 \mathrm{G}$ ), or that no highly efficient $B$-amplification is taking place in S1, then the NT emission is completely dominated by $\mathrm{S} 2$ (i.e. $B_{2}$ is the only relevant magnetic field). Fitting the observed energy fluxes requires that in S2 the magnetic field energy density is $38 \%$ of the thermal energy density, which corresponds to a magnetic field intensity of $B_{2} \sim 150 \mathrm{mG}$, and $\eta_{\text {acc }} \sim 10^{-7}$. For typical values of the Alfvén radius $r_{\mathrm{A}}=R_{2}$ and rotational speed $V_{\text {rot }}=250 \mathrm{~km} \mathrm{~s}^{-1}$, this yields a maximum value for the stellar surface magnetic field $B_{* 2}<800 \mathrm{G}$; this condition is relaxed if magnetic field amplification is taking place in the shocks. Figure 2 shows a good agreement between our model and the new radio observations. Features of the non-simultaneous observations are also reproduced qualitatively, although it is not clear if the cut-off at lower energies can be explained by free-free absorption in the stellar wind (suppression due to Razin-Tsytovich effect is negligible due to the high value of $B$; we further discuss this in Sect. 4.3).

In the MR model, the acceleration time depends heavily on $B$. In $\mathrm{S} 1$ no efficient acceleration takes place, while in $\mathrm{S} 2$ electrons can reach energies up to $\sim 100 \mathrm{GeV}$, this being the maximum energy limited by IC losses with stellar photons.
Table 2. Parameters of the WR 146 system for the primary (sub-index 1) and the secondary (sub-index 2).

\begin{tabular}{|c|c|c|}
\hline Parameter & Value & Unit \\
\hline Primary spectral type & WC6 & \\
\hline Secondary spectral type & $\mathrm{O} 8$ & \\
\hline Distance & $d=1.2$ & $\mathrm{kpc}$ \\
\hline System separation & $D_{\text {proj }}=160$ & mas \\
\hline Inclination of the orbit & $i=30$ & 0 \\
\hline Period & $P=3.38$ & $\mathrm{yr}$ \\
\hline Wind momentum ratio & $\eta=0.1$ & \\
\hline$T_{\text {eff } 1}$ & 49000 & $\mathrm{~K}$ \\
\hline$R_{1}$ & 1.5 & $R_{\odot}$ \\
\hline (primary) & 2900 & $\mathrm{~km} \mathrm{~s}^{-1}$ \\
\hline$\dot{M}_{1}$ & $5.5 \times 10^{-5}$ & $M_{\odot} \mathrm{yr}^{-1}$ \\
\hline$V_{\text {rot }_{1}} / v_{\infty_{1}}$ & $\sim 0.1$ & \\
\hline$\mu_{\mathrm{w} 1}$ & 5.29 & \\
\hline$T_{\mathrm{w} 1}$ & 8000 & K \\
\hline$T_{\text {eff2 }}$ & 32000 & $\mathrm{~K}$ \\
\hline$R_{2}$ & 10 & $R_{\odot}$ \\
\hline (secondary) & 1600 & $\mathrm{~km} \mathrm{~s}^{-1}$ \\
\hline$\dot{M}_{2}$ & $8 \times 10^{-6}$ & $M_{\odot} \mathrm{yr}^{-1}$ \\
\hline$V_{\text {rot }_{2}} / v_{\infty_{2}}$ & $\sim 0.1$ & \\
\hline$\mu_{\mathrm{w} 2}$ & 1.4 & \\
\hline$T_{\mathrm{w} 2}$ & $0.4 T_{\text {eff } 2}$ & $\mathrm{~K}$ \\
\hline
\end{tabular}

Notes. $\mu$ is the mean atomic weight and $v_{\infty}$ is the wind terminal velocity. For further information on these system parameters, refer to Setia Gunawan et al. (2000) and Dougherty \& Williams (2000).

The expected IC luminosity is not very high and falls below the gamma-ray detector sensitivities of the LAT on board the Fermi satellite (4-yr of integration time) and the Cherenkov Telescope Array (CTA, 50-h).

\subsection{Depolarization processes}

Synchrotron radiation can be depolarized by various radiation transfer effects. In transparent radio sources the main effect is Faraday rotation (FR). FR alters the plane of polarization and can reduce the observed PD. Sokoloff et al. (1998) reviewed the different mechanisms that produce depolarization of synchrotron radio emission. In what follows we will analyze the most relevant of these processes in CWBs, besides the one presented above. To be more conservative, we assume a classical DSA scenario. In such case, the available energy to accelerate NT particles is larger, as it is provided by the wind kinetic energy instead of the magnetic field. Moreover, the acceleration timescale in the fast shocks is shorter than in the MR scenario. To fit the observed synchrotron fluxes, a value of $B_{2} \sim 40 \mathrm{mG}$ is then sufficient.

1. Reduced polarization due to thermal fraction: a significant contribution from thermal (unpolarized) emission can reduce the observed PD. However, the thermal contribution in $L$-band is below $1 \%$ of the total emission, while in $C$-band it is lower than $5 \%$. This effect can therefore be neglected.

2. Differential (depth) FR: when synchrotron emission originates in a thermal plasma containing a regular magnetic field, the polarization plane of the radiation produced at different 
depths within the source is rotated over different angles by the Faraday effect. The emission from deeper regions in the volume is Faraday rotated along the line of sight, and cancels out the polarization from the surface layers of the volume, resulting in a decrease in the $\mathrm{PD}$ of the integral emission observed (e.g. see Velusamy \& Kundu 1975, for a study of this effect in supernova remnants). This is a wavelengthdependent depolarization process that is likely to require a more laminar flow than present in the WCR. In the case of WR 146, the large separation between the stars means that the shocks in the WCR will be adiabatic, and therefore reasonably thin (although a high level of turbulence could widen them). The shock width can be estimated as $\sim 0.1$ times its distance to the respective star, yielding $\Delta l_{1} \approx 20 \mathrm{AU}$ and $\Delta l_{2} \approx 5 \mathrm{AU}$. Typical values for the physical parameters at the shocks in the WCR are an electron number density of $n_{\mathrm{e} 1} \sim 10^{5} \mathrm{~cm}^{-3}$ and $n_{\mathrm{e} 2} \sim 10^{6} \mathrm{~cm}^{-3}$, and a magnetic field of $B_{1} \sim 3 \mathrm{mG}$ and $B_{2} \sim 40 \mathrm{mG}$. Considering the simplest case of a uniform slab, we can construct a rough estimate of the maximum intrinsic RM $\sim K_{\mathrm{RM}} n_{e} B_{z} \Delta l$, where the constant $K_{\mathrm{RM}}$ is equal to $81 \mathrm{~cm} \mathrm{pc}^{-1} \mathrm{G}^{-1}$ and $B_{z} \sim B / \sqrt{3}$ is the component of the magnetic field in the direction of the line of sight (LOS). We obtain values of $\mathrm{RM}_{1} \sim 5 \times 10^{3} \mathrm{rad} \mathrm{m}^{-2}$ and $\mathrm{RM}_{2} \approx 30 \mathrm{RM}_{1}$. The depolarization factor in this case is $\xi_{2}(\lambda) \approx \sin \left(\mathrm{RM}_{2} \lambda^{2}\right) /\left(\mathrm{RM}_{2} \lambda^{2}\right)<0.3 \%$ for $\lambda>5 \mathrm{~cm}$, which shows that this effect could be relevant in the case of a dominant ordered magnetic field. A detailed analysis of this process will be addressed in a forthcoming work, with an improved modeling of the quantities $n_{\mathrm{e}}$ and $B_{z}$ along the LOS through the shock.

3. Internal Faraday Dispersion: in the WCR the synchrotron emitting electrons and thermal plasma coexist. In the case the latter is turbulent, nearby lines of sight experience a random walk in FR. If the telescope beam encompasses many turbulent cells, the observed PD diminishes. This is a wavelength-dependent process. Using the simple formulas given by Burn (1966) for the case of a uniform slab, one has $S=2\left(K_{\mathrm{RM}} n_{\mathrm{e}} B_{\mathrm{r}}\right)^{2}(\delta L)^{2} \lambda^{4} \sim 10^{-2} \mathrm{RM}^{2} \lambda^{4}$, where we assumed $\delta L \sim 0.1 \Delta L$ and $B_{\mathrm{r}} \sim B_{z}$. This yields $\xi_{2}(5 \mathrm{~cm}) \approx$ $\left(1-\mathrm{e}^{-S}\right) / S<0.1 \%$. Once again, this very simple approximation just shows that the depolarization could be strong, although a more careful analysis is needed.

4. External Faraday Dispersion: this is similar to the internal effect above, but in this case the FR takes place in a thermal plasma (the stellar wind) external to the synchrotron emitting volume (the WCR). To properly account for this process, one has to solve numerically the value of $\mathrm{RM}=K \lambda^{2} \int n B_{z} \mathrm{~d} z$ for each emitting region in the WCR. This integral depends on both the intensity and the geometry of the stellar magnetic field. In the case of a toroidal magnetic field (which is expected at large distances from massive stars) supported by a surface stellar magnetic field of $B_{*} \sim 200 \mathrm{G}$, the value of $\mathrm{RM}$ can be in the range $(3-40) \times 10^{4} \mathrm{rad} \mathrm{m}^{-2}$, with significant dependence on the LOS for the particular surface element. However, this condition can be considerably relaxed if the stellar magnetic field is much lower; in particular, the strength of $B_{2}$ could be due to a high local amplification. Other external Faraday screens in the interstellar medium are most likely negligible on the angular scales of interest here.

5. Gradients in RM across the beam: this is similar to the dispersion effects above, but here describes variations in $\mathrm{RM}$ originating from systematic gradients within the telescope beam rather than from a random walk. In our case, we are looking at a curved shock that is completely unresolved within the beam of our observations. Then, if there is a gradient in RM along the shock, polarization vectors within the beam may cancel, leading to depolarization. However, one would expect that this effect does not lead to a complete depolarization of the emission as long as the LOS does not coincide with the axis of symmetry of the WCR (which would seldom occur and only if the orbit is seen edgeon at inclination $\sim 90^{\circ}$ ). Future high resolution (VLBI) polarimetric observations may be used to examine this effect. Spatially resolved fractional polarization measurements of the shock could be used to discriminate between two cases: (i) the emission appears polarized, in which case the acceleration mechanism is likely DSA due to coherent $B$-fields; or (ii) the emission is still depolarized, in which case either MR or external depolarization could be responsible.

\subsection{Source variability and possible contamination}

The WR 146 system is a variable radio source (Setia Gunawan et al. 2000). Orbital modulation could play a significant role in its variability if the eccentricity and/or the inclination of the orbit are large (Eichler \& Usov 1993). If the orbit is eccentric, conditions in the shocked plasma will vary, as will the medium in which the synchrotron photons propagate. If the orbital inclination is large, this will affect the degree to which low-frequency photons emitted in the WCR will be absorbed within the stellar wind, depending on the photon path to the observer and the orbital phase. If the binary is seen approximately edge-on $\left(i \sim 90^{\circ}\right)$, the absorption will be maximum when one of the stars is in front of the WCR (conjunction) and minimum when they are near quadrature. However, as VLBI observations of the system have revealed a C-shaped WCR (O'Connor et al. 2005), this points to a rather small orbital inclination, unless those observations of the binary correspond to rather specific positions of the orbit near quadrature (if the system were near conjunction, the WCR would look like more circular; e.g. see Fig. 4 in del Palacio et al. 2016). To explain the steep low-frequency cutoff in the spectrum seen in Fig. 2, there are two other possibilities: (i) the effect is mostly due to the eccentricity of the orbit, in which case both observations at $325 \mathrm{MHz}$ should correspond to orbital phases different to those of the observations at $5 \mathrm{GHz}$ (some coincidence is demanded, as the observations were performed at random phases); or (ii) the low-frequency cutoff is related to the Razin-Tsytovich effect, which produces a cutoff at $v_{\mathrm{RT}} \sim 20 n_{\mathrm{e}} / B \mathrm{~Hz}$ (in c.g.s. units). Given that typically $n_{\mathrm{e} 2} \sim 10^{6}$ in $\mathrm{S} 2$, this requires $B_{2} \sim 50 \mathrm{mG}$. This value of $B_{2}$ is too small for MR, and therefore the cutoff is more consistent within a DSA scenario. Nevertheless, we remark that our interpretation of the low-frequency cutoff solely in terms of free-free absorption is subject to the unknown orbital parameters; simultaneous observations at low $(<1 \mathrm{GHz})$ and high $(>1 \mathrm{GHz})$ frequencies would help to disentangle this ambiguity.

Moreover, it is still under debate whether the secondary in WR 146 is an O8V star or another binary (Setia Gunawan et al. 2000). Despite this uncertainty in the composition of the secondary, the very high resolution image from O'Connor et al. (2005) shows signs of only one WCR, so it seems unlikely that our results are biased by the behavior of the secondary. Instead, the most reasonable explanation is that there is only one dominant WCR whose variability (especially at low frequencies) is due to geometrical modulations. Long-term monitoring is needed to confirm this. 


\section{Conclusions}

We presented the first radio polarimetric study of a collidingwind binary. Our VLA observations of WR 146 reveal the absence of linearly polarized emission down to a fractional level of $0.6 \%$ across 1 to $8 \mathrm{GHz}$. If acceleration were due predominantly to DSA in strong shocks, as usually assumed, then the magnetic field component parallel to the shock front would be enhanced, and the resultant emission would be polarized. The absence of polarization can be explained in terms of a highly turbulent magnetic field, in which case turbulent magnetic reconnection would account for non-thermal particle acceleration, or depolarization effects, for example due to Faraday rotation in the stellar wind, or perhaps a combination of these effects. We have shown that, in principle, either scenario is plausible. If turbulent MR is taking place, a high value of the magnetic field in the WCR $\sim 150 \mathrm{mG}$ is required to account for the synchrotron emission. On the other hand, if DSA is the main acceleration mechanism, a magnetic field of $\approx 40 \mathrm{mG}$ is sufficient to account for the synchrotron emission. However, even this relatively low magnetic field was shown to be enough to produce significant FR and consequently strong depolarization. Moreover, a lower value of the magnetic field in the WCR also helps to reproduce the low-frequency cutoff seen in the spectrum, as in this case the Razin-Tsytovich suppression is more effective. Our analysis seems to favor a scenario where DSA and a partially ordered $B$-field are responsible for the synchrotron emission, while various depolarization processes account for the very low PD in the radiation. Future high resolution observations of the shock could be used to reveal polarized emission, where the angular scale at which the shock appears polarized would be an important diagnostic discriminant between MR and DSA. An RM detection would also help to distinguish between MR and DSA by providing constraints on magnetic field strengths, and thus checking if MR remains plausible. In a forthcoming work we will investigate the depolarization effects in more detail, and study if the detection of polarization remains elusive in other particle-accelerating colliding-wind binaries.

Acknowledgements. P.B. and G.E.R. are members of the CIC, CONICET G.E.R. acknowledges support by the Spanish Ministerio de Economía y Competitividad (MINECO/FEDER, UE) under grant AYA2016-76012-C3-1-P and CONICET grant PIP 0338. The National Radio Astronomy Observatory is a facility of the National Science Foundation operated under cooperative agreement by Associated Universities, Inc.

\section{References}

Benaglia, P., \& Romero, G. E. 2003, A\&A, 399, 112

Bieging, J. H., Abbott, D. C., \& Churchwell, E. B. 1989, ApJ, 340, 518

Bosch-Ramon, V., \& Rieger, F. M. 2012, in Astroparticle, Particle, Space Physics and Detectors For Physics Applications - Proc. of the 13th ICATPP Conf. (World Scientific Publishing Co. Pte. Ltd.), eds. S. Giani, et al., 219

Brentjens, M. A., \& de Bruyn, A. G. 2005, A\&A, 441, 1217

Burn, B. J. 1966, MNRAS, 133, 67

Cioffi, D. F., \& Jones, T. W. 1980, AJ, 85, 368

Cotton, W. D., Dallacasa, D., Fanti, C., et al. 1997, A\&A, 325, 493

De Becker, M., \& Raucq, F. 2013, A\&A, 558, A28

del Palacio, S., Bosch-Ramon, V., Romero, G. E., \& Benaglia, P. 2016, A\&A, 591, A139

del Valle, M. V., \& Romero, G. E. 2012, A\&A, 543, A56

del Valle, M. V., Romero, G. E., Luque-Escamilla, P. L., Martí, J., \& Ramón Sánchez-Sutil, J. 2011, ApJ, 738, 115

Dougherty, S. M., \& Williams, P. M. 2000, MNRAS, 319, 1005

Dougherty, S. M., Williams, P. M., van der Hucht, K. A., Bode, M. F., \& Davis, R. J. 1996, MNRAS, 280, 963

Drury, L. O. 2012, MNRAS, 422, 2474

Eichler, D., \& Usov, V. 1993, ApJ, 402, 271

Falceta-Gonçalves, D., \& Abraham, Z. 2012, MNRAS, 423, 1562

Felli, M., \& Massi, M. 1991, in Wolf-Rayet Stars and Interrelations with Other Massive Stars in Galaxies, eds. K. A. van der Hucht, \& B. Hidayat, IAU Symp., 143, 87

Hales, C. A., Gaensler, B. M., Norris, R. P., \& Middelberg, E. 2012a, MNRAS, 424, 2160

Hales, C. A., Murphy, T., Curran, J. R., et al. 2012b, MNRAS, 425, 979

Jardine, M., Allen, H. R., \& Pollock, A. M. T. 1996, A\&A, 314, 594

Kashyap, V. L., van Dyk, D. A., Connors, A., et al. 2010, ApJ, 719, 900

Kowal, G., de Gouveia Dal Pino, E. M., \& Lazarian, A. 2011, ApJ, 735, 102

Lazarian, A., \& Vishniac, E. T. 1999, ApJ, 517, 700

McMullin, J. P., Waters, B., Schiebel, D., Young, W., \& Golap, K. 2007, in Astronomical Data Analysis Software and Systems XVI, eds. R. A. Shaw, F. Hill, \& D. J. Bell, ASP Conf. Ser., 376, 127

Niemela, V. S., Shara, M. M., Wallace, D. J., Zurek, D. R., \& Moffat, A. F. J. 1998, AJ, 115, 2047

O'Connor, E. P., Dougherty, S. M., Pittard, J. M., \& Williams, P. M. 2005 , in Proc. Massive Stars and High-Energy Emission in OB Associations, eds. G. Rauw, Y. Nazé, R. Blomme, \& E. Gosset, 81

Perley, R. A., \& Butler, B. J. 2013a, ApJS, 204, 19

Perley, R. A., \& Butler, B. J. 2013b, ApJS, 206, 16

Pittard, J. M., \& Dougherty, S. M. 2006, MNRAS, 372, 801

Reitberger, K., Kissmann, R., Reimer, A., Reimer, O., \& Dubus, G. 2014, ApJ, 782, 96

Romero, G. E., Benaglia, P., \& Torres, D. F. 1999, A\&A, 348, 868

Setia Gunawan, D. Y. A., de Bruyn, A. G., van der Hucht, K. A., \& Williams, P. M. 2000, A\&A, 356, 676

Sokoloff, D. D., Bykov, A. A., Shukurov, A., et al. 1998, MNRAS, 299, 189

Stevens, I. R., Blondin, J. M., \& Pollock, A. M. T. 1992, ApJ, 386, 265

Taylor, A. R., Goss, W. M., Coleman, P. H., van Leeuwen, J., \& Wallace, B. J. 1996, ApJS, 107, 239

Velusamy, T., \& Kundu, M. R. 1975, A\&A, 41, 307

Wright, A. E., \& Barlow, M. J. 1975, MNRAS, 170, 41 


\section{Appendix A: Additional table}

Table A.1 presents our VLA flux density measurements for WR 146, as displayed in Fig. 1.

Table A.1. VLA flux densities for WR 146 measured across $L$-band and

$C$-band within $64 \mathrm{MHz}$ and $128 \mathrm{MHz}$ spectral windows, respectively.

\begin{tabular}{|c|c|c|}
\hline$v[\mathrm{GHz}]$ & $S_{v}[\mathrm{mJy}]$ & $\sigma_{S_{v}}[\mathrm{mJy}]$ \\
\hline 1.040 & 95 & 0.45 \\
\hline 1.296 & 86.4 & 0.34 \\
\hline 1.360 & 84.7 & 0.21 \\
\hline 1.424 & 82.7 & 0.18 \\
\hline 1.488 & 81.6 & 0.19 \\
\hline 1.680 & 73.5 & 0.22 \\
\hline 1.744 & 73.6 & 0.2 \\
\hline 1.808 & 72.9 & 0.19 \\
\hline 1.872 & 72 & 0.19 \\
\hline 1.936 & 70.3 & 0.29 \\
\hline 2.000 & 68.4 & 0.25 \\
\hline 4.167 & 45.2 & 0.45 \\
\hline 4.295 & 44 & 0.37 \\
\hline 4.423 & 44.4 & 0.39 \\
\hline 4.551 & 42.3 & 0.33 \\
\hline 4.679 & 42 & 0.33 \\
\hline 4.807 & 41.6 & 0.38 \\
\hline 4.935 & 40.3 & 0.37 \\
\hline 5.063 & 40.2 & 0.39 \\
\hline 5.191 & 39.1 & 0.37 \\
\hline 5.319 & 38.3 & 0.32 \\
\hline 5.447 & 38.4 & 0.35 \\
\hline 5.575 & 37.7 & 0.39 \\
\hline 5.703 & 37 & 0.33 \\
\hline 5.831 & 36.3 & 0.37 \\
\hline 5.959 & 36.9 & 0.43 \\
\hline 6.039 & 36.1 & 0.43 \\
\hline 6.295 & 34.3 & 0.37 \\
\hline 6.551 & 33.3 & 0.33 \\
\hline 6.679 & 32.6 & 0.34 \\
\hline 6.807 & 32.5 & 0.35 \\
\hline 6.935 & 33.7 & 0.39 \\
\hline 7.063 & 32.1 & 0.36 \\
\hline 7.191 & 32.3 & 0.35 \\
\hline 7.319 & 32.4 & 0.35 \\
\hline 7.447 & 32.9 & 0.38 \\
\hline 7.575 & 30.7 & 0.32 \\
\hline 7.703 & 30.6 & 0.3 \\
\hline 7.831 & 30.2 & 0.32 \\
\hline
\end{tabular}

